\title{
INTERPRETATION OF THE EXERGY EQUATION FOR STEADY-FLOW PROCESSES
}

\author{
ROLAND V. SIEMONS \\ Department of Mechanical Engineering, Chair for Industrial Thermal Engineeriug, \\ Twente University of Technology, Enschede, The Netherlands
}

(Received 20 August 1984; received for publication 8 April 1985)

\begin{abstract}
We define and discuss the terms in exergy equations, with particular reference to the role of chemical terms in the exergy loss for steady-flow processes. Although there is a chemical contribution to exergy, exergy losses of steady-flow processes may be calculated by using a simple expression for the specific exergy, namely, $b=h-T^{*} s$. No restrictions are found in the material flows involved. The necessity of prescribing a standard chemical reference environment is considered and rejected. Instead, a sign convention for material flows is proposed. Finally, some results of Brzustowski's calculations are reviewed and discussed.
\end{abstract}

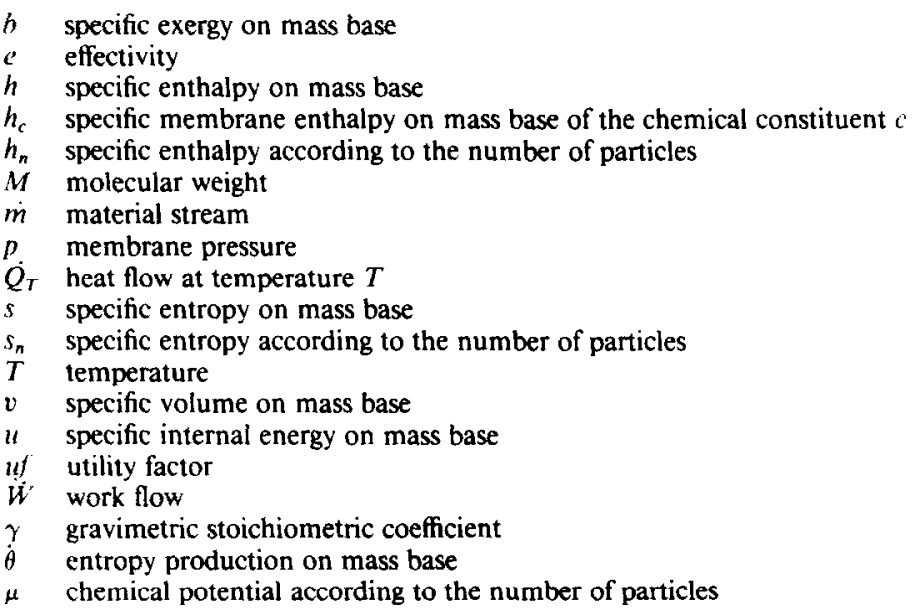

\section{NOTATION}

\section{Superscripts}

o a reversible process

at environmental conditions

\section{Subscripts}

A additional

a chemical constituent

$c, k$ a chemical constituent of substance $k$

a substance entering the system boundary

a substance leaving the system boundary

a substance either leaving or entering the system boundary temperature $T$

\section{INTRODUCTION}

We consider stationary-flow processes. In thermodynamics, these are flow processes in which the amount of every chemical element and the thermodynamic state functions of the material surrounded by a fixed control boundary are independent of time. Brzustowski' gives for these processes

$$
\begin{aligned}
\text { exergy loss }=\dot{W}+\sum_{T} \dot{Q}_{T}\left[1-\left(T^{*} / T\right)\right]+\sum_{i} & {\left[h_{i}-T^{*} s_{i}-\sum_{c} Y_{c, i}\left(\mu_{c}^{*} / M_{c}\right)\right] \dot{m}_{i} } \\
& -\sum_{j}\left[h_{j}-T^{*} s_{j}-\sum_{c} Y_{c, j}\left(\mu_{c}^{*} / M_{c}\right)\right] \dot{m_{j}}+\dagger
\end{aligned}
$$

† In Eqs. (1) and (2), the originai relations given by Brzustowski have been adapted to our signs and symbols. 
Parts of this equation are properties of the chemical constituents of matter in the environment. Brzustowski examines the effect of these chemical terms on exergy-loss computations. He distinguishes between processes in which the chemical terms cancel and those in which they do not. If they cancel ${ }^{1}$ (p. 748),

$$
\text { exergy loss }=\dot{W}+\sum_{T} \dot{Q}_{T}\left[1-\left(T^{*} / T\right)\right]+\sum_{i}\left(h_{i}-T^{*} s_{i}\right) \dot{m}_{i}-\sum_{j}\left(h_{j}-T^{*} s_{j}\right) m_{j} .
$$

Brzustowski calculates exergy losses of some examples with both equations and tries to assess the errors which are caused by disregarding the chemical terms and assumes that exergy-loss computations are correct if the chemical terms are included and incorrect if they are not. We will show why this assumption is wrong. We discuss the meaning of chemical terms in exergy calculations, clarify the utility of chemical reference environments in exergy calculations and, finally, review some results of Brzustowski's work.

\section{THERMODYNAMIC FUNDAMENTALS}

Stationary-flow processes can be studied by means of a fixed control boundary. A control boundary with the material, heat and work flows is shown in Fig. 1. The material streams which enter and leave the system boundary are distinguished by the indices $i$ and $j$. Arrows define the positive directions. That part of the body which is surrounded by the control boundary is called the system. Parts of the body are infinitesimal masses $\mathrm{d} m_{i}$ and $\mathrm{d} m_{j}$. During the time $\mathrm{d} t$, they enter or leave the control boundary. At time $t$, the internal energy of the body is

$$
U_{i}=U_{\text {system }}+\sum_{i} u_{i} \mathrm{~d} m_{i}
$$

At time $t+\mathrm{d} t$

$$
U_{t+\mathrm{d} t}=U_{\mathrm{system}}+\sum_{j} u_{j} \mathrm{~d} m_{j}
$$

The increase is

$$
\mathrm{d} U=\sum_{j} u_{j} \mathrm{~d} m_{j}-\sum_{i} u_{i} \mathrm{~d} m_{i}
$$

Use has been made of the fact that the process is stationary. For this increase, the first law states

$$
\mathrm{d} U=\sum_{T} \mathrm{~d} Q_{T}+\mathrm{d} W+\sum_{i} p_{i} v_{i} \mathrm{~d} m_{i}-\sum_{j} p_{j} v_{j} \mathrm{~d} m_{j}
$$

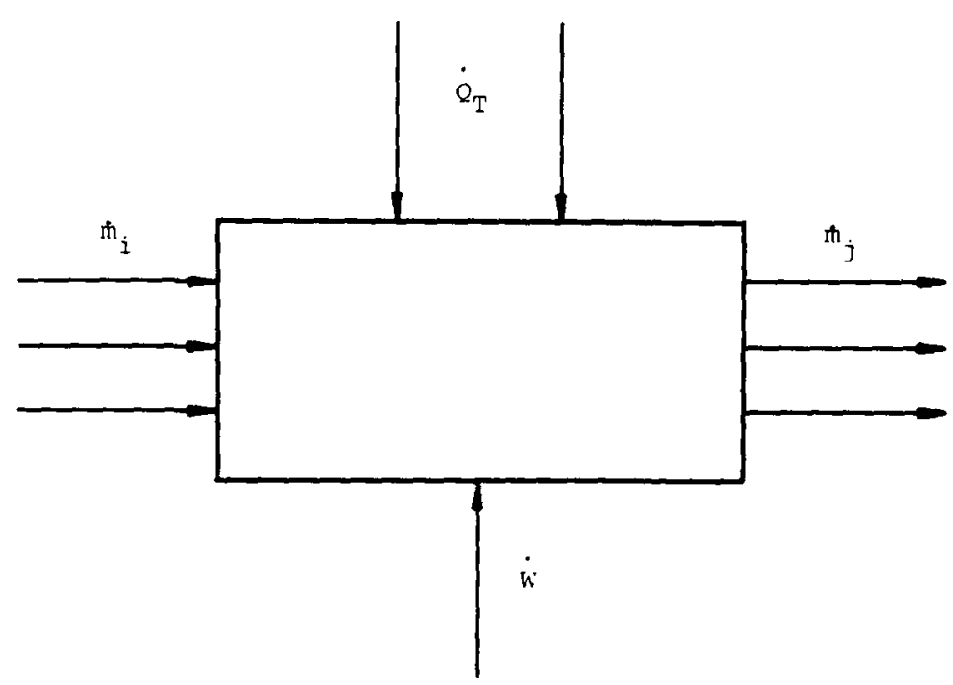

Fig. 1. Control boundary with material, heat and work flows. 
From Eqs. (3) and (4) and the definition $h=u+p v$, it follows that

$$
\sum_{T} \dot{Q}_{T}+\dot{W}+\sum_{i} h_{i} \dot{m}_{i}-\sum_{j} h_{j} \dot{m}_{j}=0
$$

The second law is

$$
\mathrm{d} S=\sum_{T} \mathrm{~d} Q_{T} / T+\mathrm{d} \theta \cdot \dagger
$$

We obtain similarly

$$
-\sum_{i} s_{i} \dot{m}_{i}+\sum_{j} s_{j} \dot{m}_{j}-\sum_{T} \dot{Q}_{T} / T=\dot{\theta}
$$

where $\theta$ represents the production of entropy by irreversabilities. Because the entropy of the system does not change in a stationary process, $\dot{\theta}$ represents the increase of the environmental entropy. Multiplying Eq. (6) by the lowest available reservoir temperature $T^{*}$ and adding to Eq. (5), we obtain

$$
T^{*} \dot{\theta}=\dot{W}+\sum_{T} \dot{Q}_{T}\left[1-\left(T^{*} / T\right)\right]+\sum_{i}\left(h_{i}-T^{*} s_{i}\right) \dot{m}_{i}-\sum_{i}\left(h_{j}-T^{*} s_{j}\right) \dot{m}_{j}
$$

The r.h.s. of Eq. (7) is the same as that of Eq. (2). In Eq. (7), only the states of the body on the control boundary are considered, not the actual process occurring in the system. Therefore, this equation is also valid when chemical reactions are involved, as in combustion processes. With this equation, we have a tool for second-law analysis of stationary-flow processes.

\section{THE MEANING OF CHEMICAL EXERGY TERMS}

The meaning of the chemical terms in Eq. (1) is clarified by deriving the equation for the maximum amount of work that can be obtained when bringing a material stream $\dot{m}_{k}$ into environmental cquilibrium. This equilibrium includes chemical equilibrium. By definition, this amount of work is the exergy of the material stream.

The maximum amount of work is obtained when the process is reversible. We will not consider the process but only the state of the material stream entering the system boundary and that of the chemical constituent streams leaving it. These states are specific enthalpy $h_{k}$ and temperature $T_{k}$ for the material stream entering and temperature $T^{*}$ and specific membrane enthalpy $h_{c}^{*}\left(h_{c}^{*}=u_{c}^{*}+p_{c}^{*} v_{c}^{*}\right)$ for the chemical constituent streams leaving. A steady-flow process will be considered. The only heat reservoir is the environment. Positive directions are again defined by the arrows in Fig. 2. It is a stationary-flow process, so that Eq. (7) applies. From Eq. (7),

$$
-\dot{W}_{k}^{o}=\left(h_{k}-T^{*} s_{k}\right) \dot{m}_{k}-\sum_{c}\left(h_{c, k}^{*}-T^{*} s_{c, k}^{*}\right) \dot{m}_{c, k}
$$

Here, use has been made of the fact that the temperature of the heat flow $\dot{Q}^{\circ}$ is that of the environment, and that the process is reversible $(\dot{\theta}=0)$.

The sum in Eq. (8) may be expressed in terms of the chemical potentials $\mu_{c}^{*}$ of the constituents under environmental conditions. Referring to the number of particles and not to the mass, the definition of the chemical potential is ${ }^{2}$

$$
\mu=h_{n}-T s_{n},
$$

so that

$$
h_{c}^{*}-T^{*} s_{c}^{*}=\left(h_{n, c}^{*}-T^{*} s_{n, c}^{*}\right) / M_{c}=\mu_{c}^{*} / M_{c} .
$$




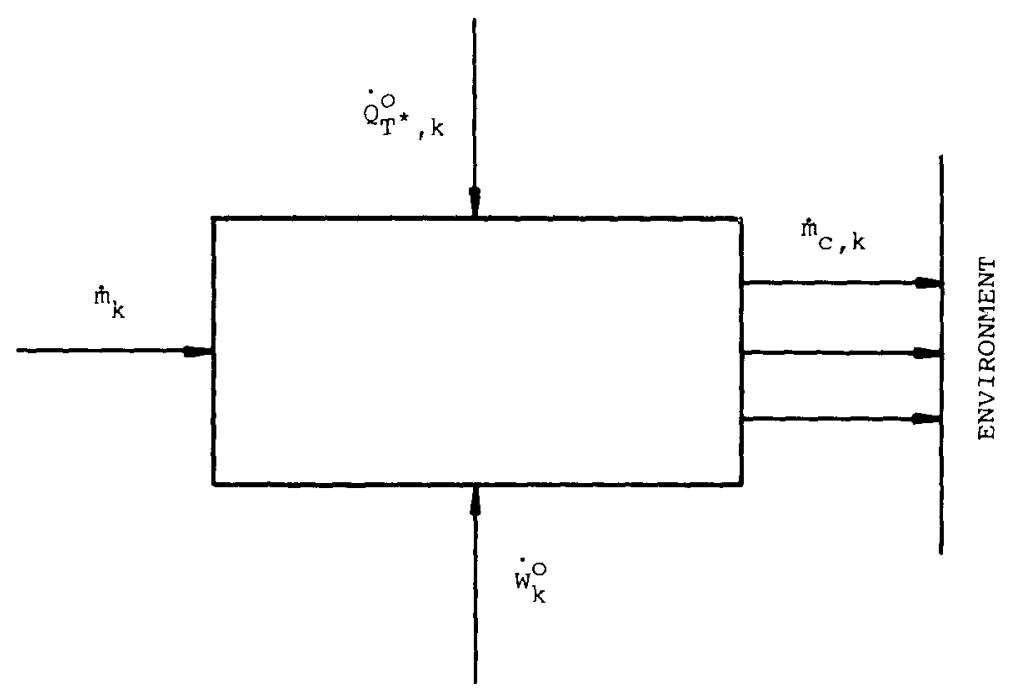

Fig. 2. Reversible stationary-flow process to bring material stream $\dot{m}_{k}$ into environmental equilibrium.

We now replace Eq. (8) by

$$
-\dot{W}_{k}^{o}=\dot{m}_{k}\left(h_{k}-T^{*} s_{k}\right)-\sum_{c} \dot{m}_{c, k}\left(\mu_{c}^{*} / M_{c}\right)
$$

One common environment belongs to various material streams $\dot{m}_{k}$. Hence $\mu_{c}^{*}$ and $M_{c}$ are independent of the matter $k$, but the streams of chemical constituents may differ for every material stream $\dot{m}_{k}$. This fact is indicated by the index $k$ in $\dot{m}_{c, k}$. With $Y_{c, k}=\dot{m}_{c, k} / \dot{m}_{k}$, we rewrite Eq. (9) as

$$
-\dot{W}_{k}^{o}=\dot{m}_{k}\left[h_{k}-T^{*} s_{k}-\sum_{c} Y_{c, k}\left(\mu_{c}^{*} / M_{c}\right)\right]
$$

Because of this result, the specific exergy of a material stream may be defined as

$$
b=h-T^{*} s-\sum_{c} Y_{c}\left(\mu_{c}^{*} / M_{c}\right)
$$

For chemical systems, Evans ${ }^{3}$ has shown that $b$ is the most general measure of exergy. In order to determine the specific exergy, the environment, i.e. $T^{*}$ and $\mu_{c}^{*}$, must be specified. According to Kestin ${ }^{4}$ chemical environmental equilibrium may or may not be constrained. His only requirement is that the environmental equilibrium must be a dead state, which means that environmental chemical potentials must be independent of time. In Sec. 4, we show why this condition is insufficient.

Making use of Eq. (11) in an exergy balance (exergy loss = exergy entering the control boundary - exergy leaving the control boundary), Eq. (1) is obtained. Equation (1) is a valid expression for the exergy loss.

\section{CANCELATION OF THE CHEMICAL TERMS}

The chemical terms in Eq. (1) must cancel, independently of the material flows that are involved in the process. We have derived two equations from first principles: Eq. (1) for the exergy loss and Eq. (7) for entropy production. Substituting Eq. (7) in Eq. (1), it follows that

$$
\begin{aligned}
\text { exergy loss } & =T^{*} \dot{\theta}-\sum_{i} \dot{m}_{i}\left[\sum_{c} Y_{c,(}\left(\mu_{c}^{*} / M_{c}\right)\right]+\sum_{j} \dot{m}_{j}\left[\sum_{c} Y_{c, y}\left(\mu_{c}^{*} / M_{c}\right)\right] \\
& =T^{*} \dot{\theta}-\sum_{i} \sum_{c}\left(\dot{m}_{c, i} \mu_{c}^{*} / M_{c}\right)+\sum_{j} \sum_{c}\left(\dot{m}_{c, j} \mu_{c}^{*} / M_{c}\right) \\
& =T^{*} \dot{\theta}+\sum_{c}\left[\left(\mu_{c}^{*} / M_{c}\right)\left(-\sum_{i} \dot{m}_{c, i}+\sum_{j} \dot{m}_{c, j}\right)\right]
\end{aligned}
$$


since $\mu_{c}^{*}$ is independent of $i$ and $j$. If no chemical reactions are involved, the flows of entering and leaving material streams are of the same chemical composition. Therefore, for every separate chemical constituent,

$$
-\sum_{i} \dot{m}_{c, i}+\sum_{j} \dot{m}_{c, j}=0
$$

With this condition,

$$
\text { exergy loss }=T^{*} \dot{\theta}
$$

Equation (12) shows that the exergy loss of a chemical process must be supplied by the second term

$$
\sum_{c}\left[\left(\mu_{c}^{*} / M_{c}\right)\left(-\sum_{i} \dot{m}_{c, i}+\sum_{j} \dot{m}_{c, j}\right)\right]
$$

However, this term is not an exergy loss but rather exergy.

The exergy of the material streams leaving the process, $\dot{m}_{j}$, is the amount of work obtained when they are brought reversibly into environmental equilibrium. After this is done, their chemical constituents may be transformed to those of the material stream entering the process, $\dot{m}_{i}$, by an additional reversible chemical process (see Fig. 3). This process is stationary because the total amount of every chemical element is conserved in the original stationaryflow process.

The additional process may require or produce work. It will be called additional work $\dot{W}_{A}^{o}$. An equation analogous to Eq. (7) will be used to derive an expression for $\dot{W}_{A}^{o}$. The additional process is reversible and its heat is exchanged at the environmental temperature; hence,

$$
\begin{aligned}
0 & =\dot{W}_{A}^{o}+\sum_{c} \sum_{j}\left(h_{c, j}^{*}-T^{*} s_{c, j}^{*}\right) \dot{m}_{c, j}-\sum_{c} \sum_{i}\left(h_{c, i}^{*}-T^{*} s_{c, i}^{*}\right) \dot{m}_{c, i} \\
& =\dot{W}_{A}^{o}+\sum_{c}\left(\mu_{c}^{*} / M_{c}\right)\left(\sum_{j} \dot{m}_{c, j}-\sum_{i} \dot{m}_{c, i}\right)
\end{aligned}
$$

and

$$
\dot{W}_{A}^{o}=\sum_{c}\left(\mu_{c}^{*} / M_{c}\right)\left(\sum_{i} \dot{m}_{c, i}-\sum_{j} \dot{m}_{c, j}\right)
$$

Equations (12) and (13) yield, for chemical processes,

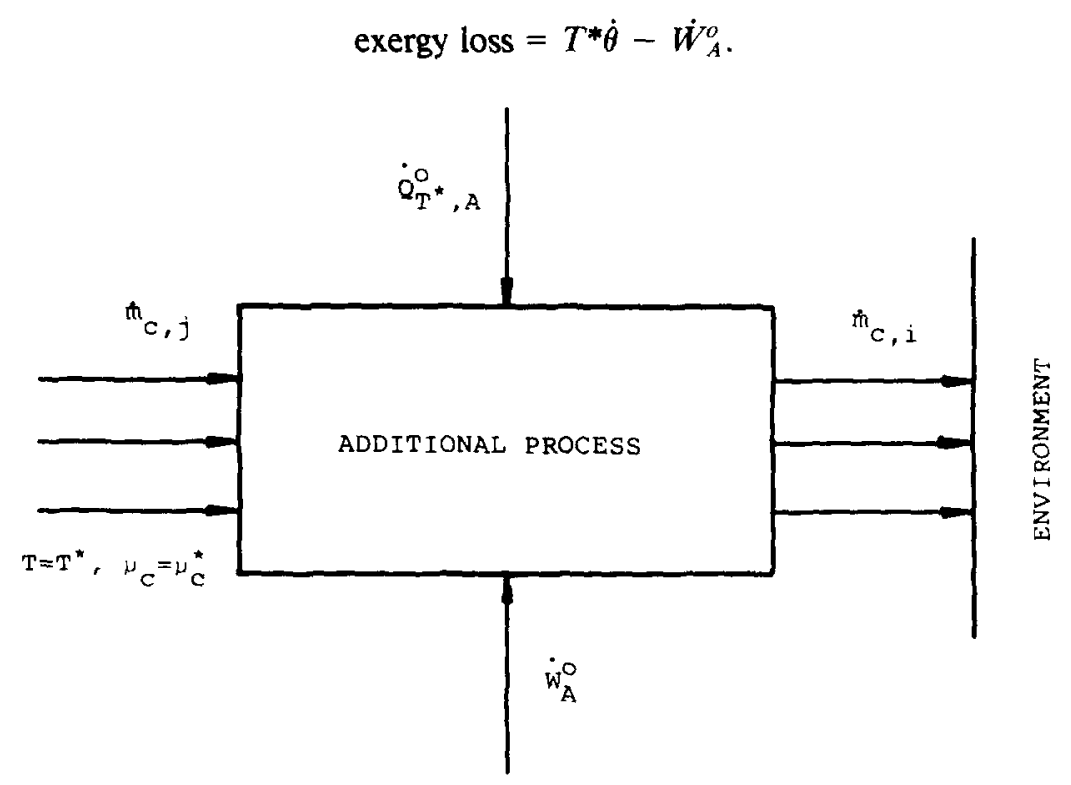

Fig. 3. Additional stationary-flow process. 
However, there is an inconsistency in the expressions for exergy and exergy loss [Eqs. (1, 11 and 12)] if the exergy loss consists partly of available work. Therefore, the additional work must be zero.

If the proportions of the chemical constituent streams entering the additional process are not stoichiometric, some amounts of the constituents will not take part in the reaction but will instead leave the additional process unchanged. These parts of the constituent streams cancel in Eq. (13). The rest of the chemical constituent streams can be related to one particular constituent stream by means of gravimetric stoichiometric coefficients. Thus, from Eq. (13),

$$
-\dot{W}_{A}^{o}=\dot{m}_{c 1} \sum_{c}\left(\mu_{c}^{*} / M_{c}\right) \gamma_{c}
$$

where the subscript $c 1$ denotes a particular chemical component. The additional work can only equal zero if

$$
\sum_{c}\left(\mu_{c}^{*} / M_{c}\right) \gamma_{c}=0
$$

which means that the environmental reservoir is in unconstrained equilibrium. ${ }^{5}$

As a consequence, the chemical terms in Eq. (1) cancel and

$$
\text { exergy loss }=T^{*} \dot{\theta}
$$

whether the process involves chemical reactions or not. This remarkable fact has also been shown by Ahrendts. ${ }^{6,7}$ Our treatment differs from that of Ahrendts since we stipulate unconstrained equilibrium for consistency of concepts and expressions for exergy and exergy loss.

We have shown that the r.h.s. of Eq. (1) has been reduced to that of Eq. (7). We may conclude that the exergy loss equals $T^{*} \dot{\theta}$ and that Eq. (2) is not an approximation of Eq. (1).

For combustion processes, Brzustrowski ${ }^{1}$ (pp. 748, 753 et seq.) suggests that cancelling of the chemical terms depends on whether gases are dissociated or not, or on whether the air-fuel ratio is stoichiometric or not, i.e., on the material streams involved in the process. We do not share this opinion. Neither of these conditions has been used in our arguments.

\section{THE UTILITY OF A REFERENCE CHEMICAL ENVIRONMENT}

Many engineers use a simpler definition of specific exergy than that defined in Eq. (11), namely,

$$
b=h-T^{*} s
$$

The difference between the two definitions given in Eqs. (11) and (16) means a shift of the zero level of exergy. $†$ Both definitions may be useful. Engineers have to choose definitions that suit diverse purposes best. The extended definition, Eq. (11), is not necessary for the calculation of exergy losses. However, it is wrong to use Eq. (16) for one material flow and Eq. (11) for another. The exergy loss $T^{*} \dot{\theta}$ of the process will then lose its meaning.

The zero level of exergy does not influence exergy losses but is of great importance for the effectivity. The effectivity is a relative measure for the exergy loss by which various processes may be compared. Brzustowski ${ }^{\prime}$ (p. 745) defines it as

$$
e=\frac{\text { exergy leaving the system }}{\text { exergy entering the system }} \text {. }
$$


This is the same as

$$
e=\frac{\text { exergy entering the system }- \text { exergy loss }}{\text { exergy entering the system }} .
$$

The value of the exergy entering the system depends on the zero-level definition of exergy. But the exergy loss is independent of the definition used. Hence, by making use of Eq. (16) and various chemical environments, values for the calculated effectivity will differ although they belong to the same process. Kestin proposes another relative measure for exergy losses. He defines the utilisation factor $^{3}$ (p. 691) as

$$
u f=\frac{\text { actual work }}{\text { exergy entering the system }} .
$$

With this factor, we meet a similar problem. For this reason, Ahrendts ${ }^{6}$ (pp. 669 et seq.) proposes to prescribe a general chemical environment as a standard reference.

There is another problem in the use of these relative measures. Since the value of the entering exergy depends on the sign convention, the value of the effectivity also depends on this sign convention. In this paper, entering or leaving material streams are distinguished by the indices $i$ and $j$. According to this sign convention,

$$
\text { exergy entering the system }=\sum_{i} b_{i} \dot{m}_{i}+\text { terms of work and heat. }
$$

Brzustowski (p. 744) does not make this distinction. He considers the exergy of all material streams as exergy entering the system and a material stream entering the system is counted as positive, while a material stream leaving is counted as negative. Thus,

$$
\text { exergy entering the system }=\sum_{i} b_{i} \dot{m}_{i}-\sum_{j} b_{j} \dot{m}_{j}+\text { terms of work and heat. }
$$

By the same argument, effectivities or utilisation factors determined while starting from different sign conventions will differ. This argument also holds for the sign conventions of heat and mass flows. Using such relative measures for the exergy loss requires a standard sign convention.

A complication is that, in Eq. (17), all chemical terms cancel. Therefore, values of the effectivity according to different chemical environments will again be comparable. This result holds also for use with either Eqs. (16) or (11). When using the sign convention belonging to Eq. (17) for the material streams, a standard chemical environment is not necessary. For this reason, this sign convention would be suitable as standard.

\section{DISCUSSION ABOUT BRZUSTOWSKI'S RESULTS}

Making use of Eq. (16) as definition of specific exergy, Brzustowski calculates the exergy loss and effectivity for the adiabatic burning of a stoichiometric methane-air mixture. He obtains $^{1}$ (p. 749)

$$
T^{*} \dot{\theta}=235.98 \mathrm{MJ} / \mathrm{kmol} \mathrm{CH}_{4}, \quad \text { and } \quad e=0.718 .
$$

Together with Brena, Brzustowski has presented some corrections to his earlier calculations. For the same process, using the extended definition of specific exergy [Eq. (11)] and a reference environment in unconstrained chemical equilibrium, they ${ }^{8}$ find

$$
T^{*} \dot{\theta}=236.64 \mathrm{MJ} / \mathrm{kmol} \mathrm{CH}_{4}, \quad \text { and } \quad e=0.7176 .
$$

In this paper, we have shown that the values of exergy loss must be equal. The only possible conclusion is that the complicated way of calculating did not introduce large inaccuracies. Dissociation and the air-fuel ratio have nothing to do with equality of the values.

It has been stated that, in general, effectivities determined from different zero-level exergy definitions will not be equal. It is therefore remarkable that these two effectivities are equal. The reason is found in the particular sign convention used by Brzustowski. 


\section{CONCLUSION}

In technical sciences, we have to distinguish between concepts and definitions of zero levels. The concept of exergy is available work. Chemical terms belong to this concept. Engineers who are only interested in exergy losses of steady-flow processes may simply use $b=h-T^{*} s$ as a definition of the zero level of exergy. In general, effectivities based on different definitions of the zero level of exergy or on different sign conventions are not comparable. Confusion about the chemical reference can be avoided by the convention that the exergy of all material streams entering a system is taken as positive and that of all material streams leaving is taken as negative. If we do so, it is not useful to define a standard chemical environment. However, actual chemical environments may be important for real values of exergy. A standard sign convention concerning heat and work flows is still needed.

Acknowledgements-The author wishes to thank C. J. M. Den Dulk and P. De La Court for their valuable contributions. A. B. Cambel's help is also gratefully acknowledged.

1. 1. A. Brzustowski, Energy 5, 743 (1980).

2. J. Kestin, A Course in Thermodynamics. 1, 583, Blaisdell, Waltham, MA (1966).

3. R. B. Evans, "A Proof that Essergy is the Only Consistent Measure of Potential Work (for Chemical Systems)," dissertation, Dartmouth College, Hanover, NH (1969).

4. J. Kestin, Energy 5, 679 (1980).

5. J. Kestin, A Course in Thermodynamics. 2, 287, Blaisdell, Waltham, MA (1968).

6. J. Ahrendts, Energy 5, 677 (1980).

7. J. Ahrendts, "Die Exergie chemisch reaktionsfähiger Systeme. Erklärung und Bestimmung," p. 26 et seq., Dissertation, Ruhr-Universität, Bochum (1974).

8. T. A. Brzustowski and A. Brena, Energy 6, 473 (1981). 